\title{
Polarity-dependent forming in ion bombarded amorphous silicon memory devices
}

\author{
R. G. Gateru, J. O. Orwa, ${ }^{\text {a) }}$ and J. M. Shannon \\ Advanced Technology Institute, School of Electronics and Physical Sciences, University of Surrey, \\ Guildford, Surrey GU2 7XH, United Kingdom
}

(Received 18 June 2004; accepted 18 October 2004; published online 23 December 2004)

\begin{abstract}
Polarity-dependent forming in ion bombarded metal-semiconductor-metal (MSM) memory devices of hydrogenated amorphous silicon is reported. It is shown that prior to ion bombardment, current transport in the MSM devices is asymmetric and is controlled by the Schottky barriers at two MS junctions. Upon bombardment, however, there is a bulk component to the current and the $I-V$ characteristics of the devices become symmetric at low bias voltages. The forming voltage in the bombarded devices shows polarity dependence. For positive bias applied on the top contact, we find that devices form at the same electric field independent of the thickness of the amorphous silicon while for negative voltage on the top contact, the electric field needed for forming increases with the thickness. A model involving the difference in energy deposition and heat sinking for the two polarities is proposed. (C) 2005 American Institute of Physics. [DOI: 10.1063/1.1832748]
\end{abstract}

\section{INTRODUCTION}

Memory switching is said to occur when a solid-state device [metal-semiconductor-metal (MSM) in this case] changes from a highly nonconducting (OFF) state to a highly conducting $(\mathrm{ON})$ state accompanied by a change in the device resistance over several orders of magnitude. This change remains stable even in the absence of any bias, making these devices nonvolatile (memory) switching devices-as opposed to threshold switching devices in which the ON state is maintained only in the presence of a bias. The initial stage when as-deposited devices are switched to the ON state for the first time is referred to as forming. Though the actual mechanisms of forming (and subsequent switching) are still unknown, it is thought that a highly conducting semimetallic filament is created through the device during forming, ${ }^{1}$ creating a link between the two metal contacts which produces the large reduction in the device resistance in the ON state. Subsequent switching then occurs by the breaking and recreating of this filament with pulses of lower magnitude than those required for the initial forming process. Hajto $e t \mathrm{al}^{2}$ give a detailed review of most of the characteristics associated with both threshold and memory switching, including forming. Work on reversible switching has been carried out on a number of materials, starting originally with the chalcogenide glasses, ${ }^{3}$ to nickel oxides, ${ }^{4}$ amorphous silicon, ${ }^{5}$ hydrogenated amorphous silicon, ${ }^{6}$ and more recently in hydrogenated amorphous (silicon-rich) silicon carbide. ${ }^{7,8}$ The forming step is a rather difficult process to control but it has been observed that slightly doping the semiconductor film significantly lowers the forming voltage. This is thought to be related to the presence of defect states introduced into the semiconductor film by the doping process. ${ }^{9}$ In this work, silicon dangling-bond defect states have been introduced into the semiconductor material by

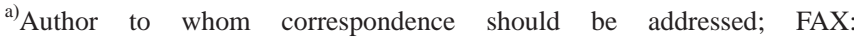

+44(0)1483689404; electronic mail: j.orwa@surrey.ac.uk
}

bombarding the film with $\mathrm{Si}^{+}$ions. Ion bombardment has distinct advantages in that the distribution profile and the density of the induced defects can be controlled with much more ease compared with the previous methods of doping ${ }^{9}$ and current stressing. ${ }^{10}$ This control is achieved by selecting appropriate implantation parameters such as dose and energy, and it has been observed that ion bombardment of the MSM structures results in more uniform electrical and forming characteristics. ${ }^{8,11}$ We use $\mathrm{Si}^{+}$ions in order to ensure that mainly silicon dangling-bond states are introduced without doping the material. The Si dangling-bond defect states will tend to pin the Schottky barriers at both MS junctions at a well-defined level, as well as control the minority-carrier lifetime and Poole-Frenkel conduction, ${ }^{12}$ all of which are instrumental in determining how the current flows and the energy is dissipated in the MSM structure during forming and switching.

\section{EXPERIMENTAL DETAILS}

Ion bombarded hydrogenated amorphous silicon $(a-\mathrm{Si}: \mathrm{H}) \mathrm{MSM}$ devices were fabricated with chromium $(\mathrm{Cr})$ top and bottom contacts. First, a chromium nitride $(\mathrm{CrN})$ back contact was deposited onto a glass substrate and the $a$ -Si:H semiconductor layer was deposited onto it by the rfglow discharge decomposition of silane $\left(\mathrm{SiH}_{4}\right)$ in a plasmaenhanced chemical-vapor deposition (PECVD) system at $300{ }^{\circ} \mathrm{C}$. The thickness of the layer was 50,70 , and $100 \mathrm{~nm}$. Ion bombardments were made using $\mathrm{Si}^{+}$ions at energies of 10,20 , and $40 \mathrm{keV}$ for the 50-, 70-, and 100-nm-thick samples, respectively. The energies were chosen so that the induced defect states were located at approximately the centers of each of the semiconductor films. Following bombardment, Cr metal contacts were sputtered onto the semiconductor film through a mask to give rectangular devices 4.23 $\times 10^{-4} \mathrm{~cm}^{2}$ in area. Forming was achieved by manually stressing the MSM devices at the top contact with unipolar single-shot pulses of mainly $4 \mu \mathrm{s}$ in length. The magnitude 


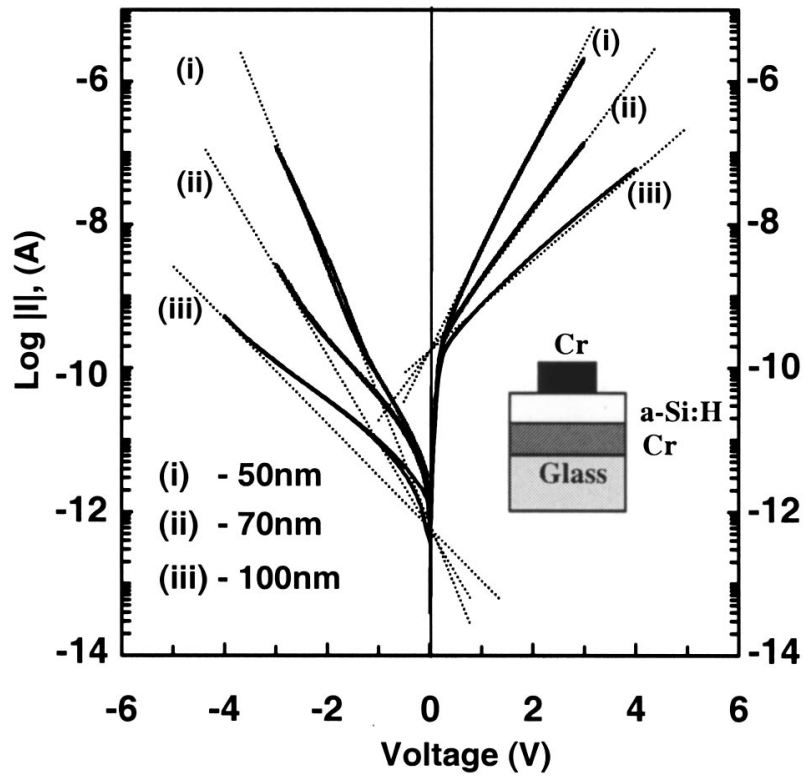

FIG. 1. Low-voltage $I-V$ characteristics for unbombarded $\mathrm{Cr} / a-\mathrm{Si}: \mathrm{H} / \mathrm{Cr}$ MSM devices.

of the forming pulses was increased from a low value $(\sim 2 \mathrm{~V})$ in increments of $0.5-1 \mathrm{~V}$ until the devices formed. $I-V$ measurements were carried out before and after each pulse stress. These were taken between $\pm 0.6 \mathrm{~V}$ in increments of $0.025 \mathrm{~V}$ at a dwell time of $0.001 \mathrm{~s}$. High-voltage $I-V$ characteristics were obtained by recording current levels at each forming voltage on a digital oscilloscope and correcting for the voltage dropped across a $1-\mathrm{k} \Omega$ resistor connected in series with the device to avoid current overshoot.

\section{RESULTS AND DISCUSSION}

Figure 1 shows the $I-V$ characteristics of the unbombarded $a$-Si:H devices of varying thicknesses. The characteristics show a large asymmetry between the positive and negative bias characteristics. This can be seen from the difference in the current levels at $V=0$ obtained by extrapolation of the characteristics. This asymmetry between the positive and negative polarities represents an asymmetry in the barrier heights at the two contacts. When a positive voltage is applied to the top contact, electrons are ejected from the bottom contact while in the case of a negative voltage on the top contact, electrons are ejected from the top contact of the MSM structure. The barrier heights $\left(\phi_{B}\right)$ at the two contacts can be estimated from the values of the intercept at $V=0$. From Fig. 1, $\phi_{\mathrm{BT}}=0.94 \mathrm{eV}$ and $\phi_{\mathrm{BB}}=0.79 \mathrm{eV}$ for the top and bottom contacts, respectively. This asymmetry in the barrier height is typical of MSM structures grown using PECVD where it is found that $a-\mathrm{Si}: \mathrm{H}$ grown on metal gives a different barrier height than when depositing a metal on $a$-Si:H. ${ }^{13}$

After ion bombardment, the $I-V$ characteristics at low fields change markedly (Fig. 2). The magnitude of the current increases and the characteristics are symmetrical at about $V=0$. This is consistent with current transport through the bulk of the material becoming more dominant compared with current transport over the barriers at each end of the devices, which is the case in the unbombarded devices. It is

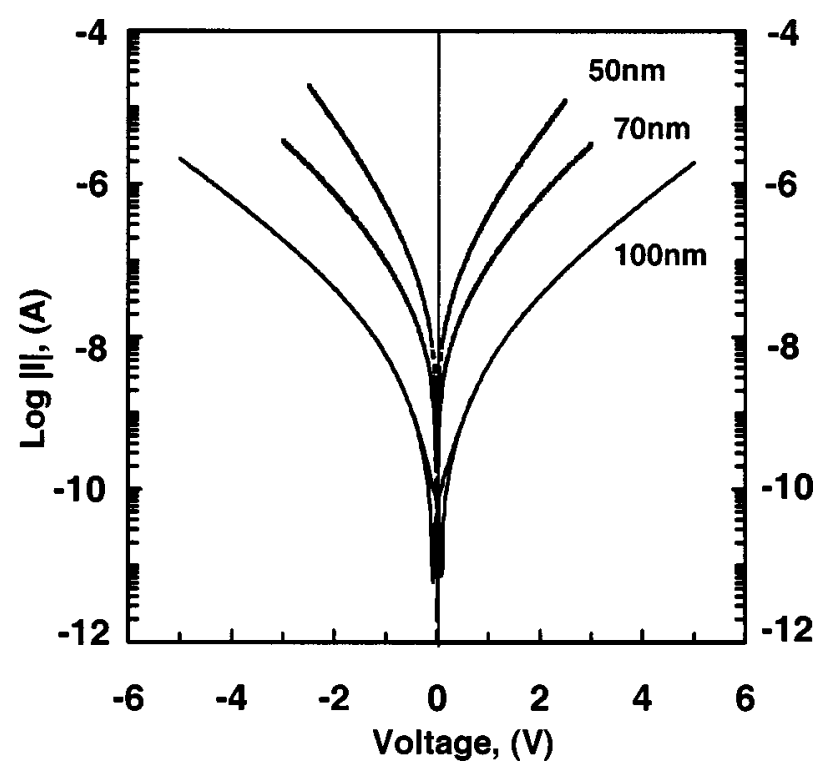

FIG. 2. Low-voltage $I-V$ characteristics for $\mathrm{Cr} / a-\mathrm{Si}: \mathrm{H} / \mathrm{Cr} \mathrm{MSM}$ devices bombarded with $5 \times 10^{12} \mathrm{Si}^{+}$ions at implant energies of 20,30 , and $40 \mathrm{keV}$, respectively.

found that the voltage required for a given current increases with the thickness of the $a-\mathrm{Si}: \mathrm{H}$ layer. The symmetry of the curves is consistent with the conduction through the bulk of the material via the Poole-Frenkel effect.

It is known that ion bombardment at room temperature introduces damage into $a-\mathrm{Si}: \mathrm{H}$, which increases the width of the band tails due to an increase in the variation of bond angles and bond lengths. ${ }^{14}$ The increase in the strength of the band tails leads to a change in the mobility edge and a reduction in the band gap. There is also a large increase in the concentration of silicon dangling-bond states distributed across the band gap. On annealing above the equilibration temperature $\left(\sim 240^{\circ} \mathrm{C}\right)$, most of these dangling-bond states anneal out but there is always some residual damage remaining that depends on the total amount of energy dissipated during the implant and, therefore, on the dose and atomic mass of the ion. ${ }^{15}$ The effect of ion bombardment, therefore, is to reduce the Schottky barrier height and provide charged defect states that enable current to flow through the surface barriers via the Poole-Frenkel effect in parallel with the current passing over the barriers via the thermionic-field emission. Since $\ln J$ is proportional to the square root of the electric field $(E)$ for the Poole-Frenkel conduction and to $\sim E$ for the thermionic-field emission, ${ }^{16}$ we expect to see the PooleFrenkel behavior at low fields and thermionic-field emission at high fields. Indeed it is possible to see the transition from one dominant transport mechanism to the other by measuring the activation energy as a function of bias. ${ }^{17}$

Figure 3 shows high-field current-voltage $(I-V)$ characteristics for both the reference (unbombarded) and bombarded MSM devices of thickness 50, 70, and $100 \mathrm{~nm}$ for both the positive and negative pulses. It can be seen that the current for the bombarded devices is higher than that for the unbombarded devices-presumably due to the effect of ion bombardment in lowering the Schottky barrier height. The current for a given field, however, is still lower in the nega- 


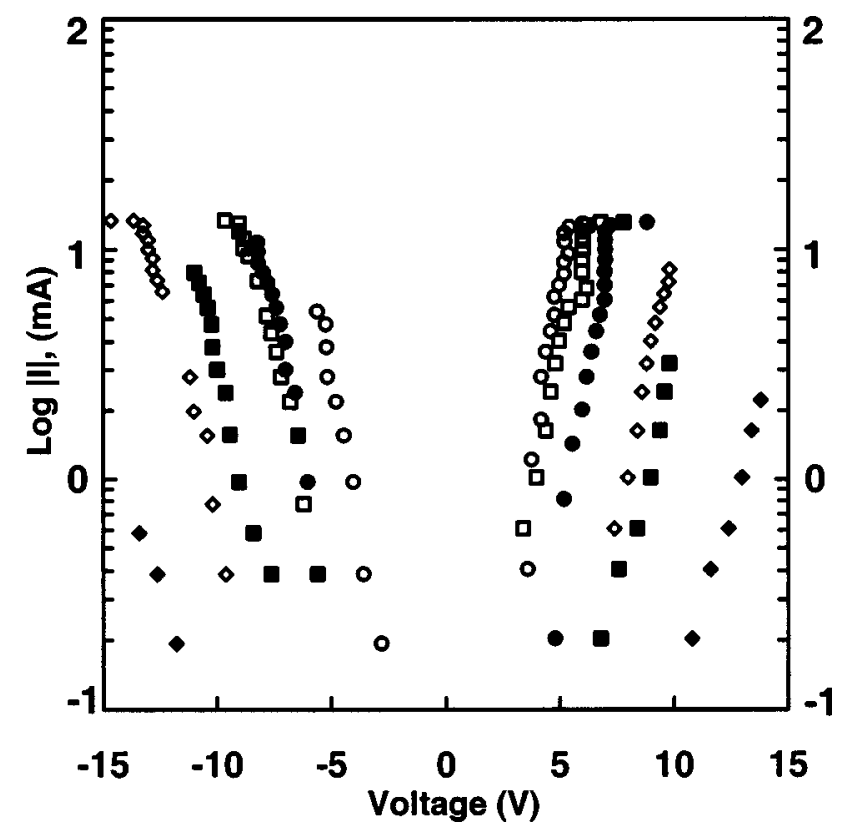

FIG. 3. High-voltage $I-V$ plots for the $(\bigcirc)$ 50-, ( $\square$ ) 70-, and $(\diamond) 100$ -nm-thick devices for both the positive and negative forming pulses. The filled symbols are for the unbombarded devices.

tive direction just as in the unbombarded reference suggesting that, although the barrier heights have been modified by the implant, some of the asymmetry between the contacts has been retained. We also note that the $E^{1 / 2}$ dependence between the logarithm of the current and electric field seen in Fig. 2 is no longer apparent. Since $E$ is proportional to $V$, Fig. 3 shows a more linear dependence between $\log I$ and $E$, indicative of thermionic-field emission. Another significant observation from Fig. 3 is that as the voltage pulses of higher magnitudes are applied to the top contact in the positive direction, there seems to be an abrupt rise in the current at $\sim 6 \mathrm{~mA}$ and the current rises almost vertically towards the current limit of the stressing kit $(\sim 13 \mathrm{~mA})$. This abrupt rise in the current could be indicative of an increase in the device temperature when the current flowing through the device exceeds $\sim 6 \mathrm{~mA}$, irrespective of the device thickness. This phenomenon is not seen in the negative direction.

Figure 4 shows the magnitude of the electric field $\left(E_{F}\right)$ required to form devices of different thicknesses. Devices located in close proximity to each other on the same 2-in. square sample were formed using the positive and negative pulses. For the positive pulses, we see that the average value of $E_{F}$ for the bombarded devices is approximately constant at $1.2 \times 10^{8} \mathrm{~V} \mathrm{~m}^{-1}$, independent of thickness. Since current is determined by thermionic-field emission through the reverse biased Schottky barrier contact and is a function only of the electric field, we assume that a similar amount of current is flowing through the devices just prior to forming. In this case, we can assume that the forming process in the positive direction is a function only of current and not power. Interestingly, the high-field $I-V$ plots shown in Fig. 3 indicate an abrupt increase in current at the same point $(\sim 6 \mathrm{~mA})$, indicative of a rise in device temperature.

For pulses with negative bias on the top contact, Fig. 4 shows that the average field required to form devices

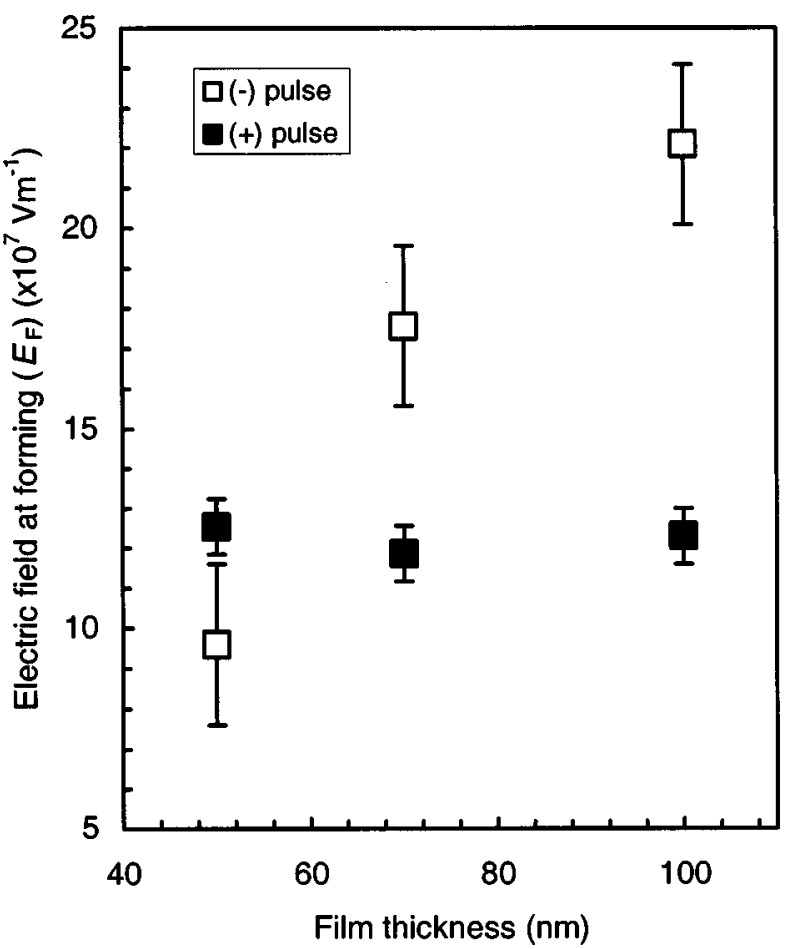

FIG. 4. Electric field $\left(E_{F}\right)$ required to form 50-, 70-, and 100-nm-thick Si-bombarded $a$-Si:H devices with positive and negative pulses on the top contact.

strongly depends on thickness of the $a$-Si:H layer, with higher fields being required for thicker layers. This seems to suggest that in the negative direction, it is power that counts, implying that higher power is required to form the thicker samples and hence the need for the higher voltages in the 70and 100-nm devices. $E_{F}$ is much higher in the thicker samples compared to the $E_{F}$ of the same samples in the positive direction.

The difference in the forming characteristics observed when the devices are stressed with the positive or negative polarities can be explained by the asymmetry in the structure and the differences between the energy deposition mechanisms at the negative and positive contacts. Since the top metal contact is a defined region while the bottom contact is continuous (see Fig. 1 inset), it is always easier to heat the top contact because it has better thermal isolation. At the bottom contact any heat dissipated during energy deposition is lost much more rapidly. We have seen that under the positive bias on the top contact, the electric field needed to form the device is independent of the thickness of the $a-\mathrm{Si}: \mathrm{H}$, suggesting that the magnitude of the electron current is all that matters. Under high electric fields, this current flows through the conduction band of the $a-\mathrm{Si}: \mathrm{H}$ and into the top metal contact [Fig. 5(a)]. Every electron that passes into the top contact will deposit $\phi_{\mathrm{BT}}$ of energy at the metalsemiconductor interface. We assume that when this energy reaches a critical level, filament formation begins. Furthermore, a high barrier to electrons at the top contact implies a low barrier to holes since their sum equals the band gap. We, therefore, expect holes to be injected at the top contact and electron-hole recombination to occur, further increasing the 

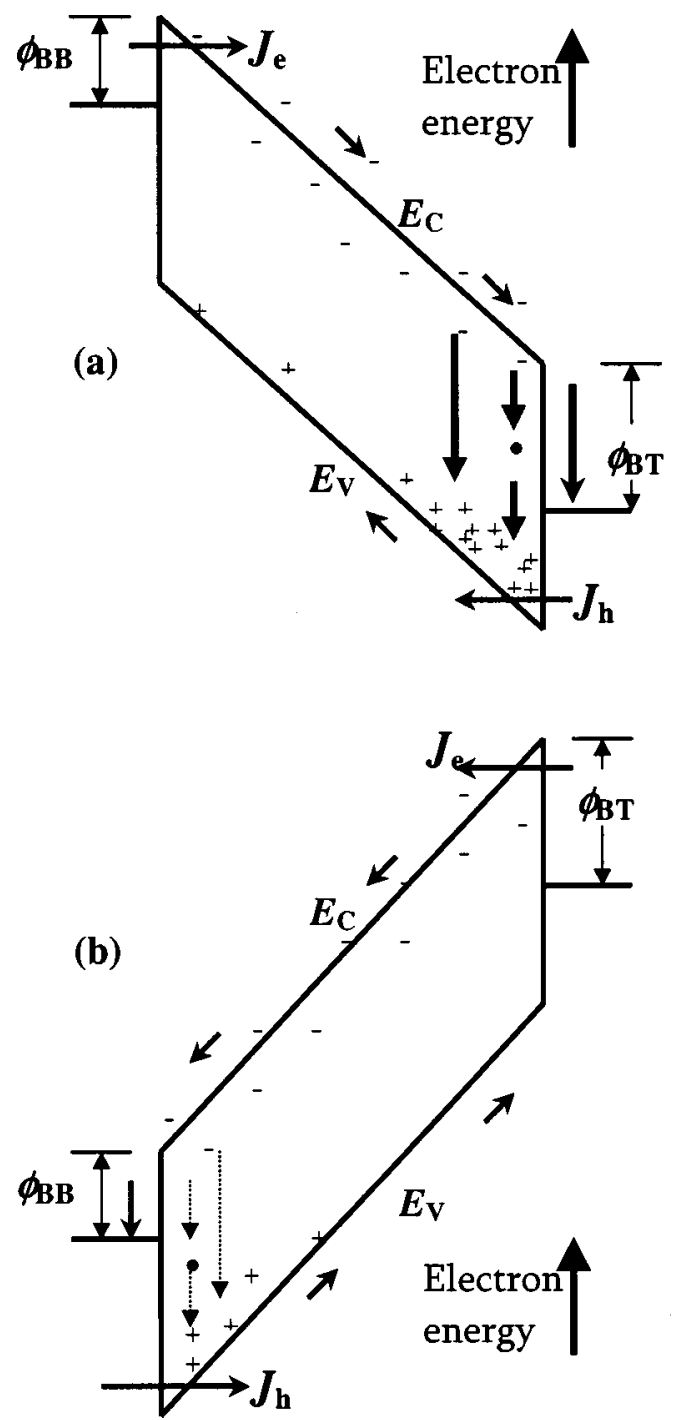

FIG. 5. Band diagram for a $\mathrm{Cr} / a-\mathrm{Si}: \mathrm{H} / \mathrm{Cr} \mathrm{MSM}$ structure with (a) positive bias and (b) negative bias on the top contact showing asymmetry in the energy deposition process. Energy deposition is much more pronounced at the top contact under the positive bias than it is under the negative bias.

energy deposition close to the positive contact. This mechanism explains why the electric field at forming is independent of the thickness of the $a-\mathrm{Si}: \mathrm{H}$.

The situation when a negative voltage is applied to the top contact is very different [Fig. 5(b)]. This is because only electrons in the metal with energy greater than that of $\phi_{\mathrm{BT}}$ flow over the barrier into the $a-\mathrm{Si}: \mathrm{H}$ thereby cooling the top contact. Energy deposition occurs throughout the $a-\mathrm{Si}: \mathrm{H}$ and particularly at the bottom contact. We see from Fig. 4 that the field required to form the thinnest devices is comparable under the positive or negative bias. This can be explained if heat generated at the bottom contact during the forming pulse is transmitted through the semiconductor to the top contact. This heat combined with that generated by dissipation in the $a-\mathrm{Si}: \mathrm{H}$ increases above the critical amount needed for forming via the top contact under the negative bias. As the thickness of the $a-\mathrm{Si}: \mathrm{H}$ increases, transmission of heat from the bottom to the top contact becomes less effective and an increase in electric field and electron current is required to increase the power dissipation in the $a-\mathrm{Si}: \mathrm{H}$ before forming occurs. We see, therefore, that the polaritydependent forming can be explained by the asymmetry in the heat sinking of the two contacts combined with a difference in the energy deposition mechanism between the positively and negatively biased contacts.

\section{CONCLUSIONS}

Measurements on bombarded MSM devices with different thicknesses have shown that forming using positive pulses is different from forming using negative pulses in terms of their field dependence. In the positive direction, the electric field needed to form a MSM device is independent of the thickness of $a-\mathrm{Si}: \mathrm{H}$. This indicates that the forming mechanism depends only on the magnitude of the current. In the negative direction, $E_{F}$ was observed to be strongly dependent on thickness with thicker samples requiring higher fields. This suggests that power, rather than the current flowing through the devices, is more important for forming with negative pulses. A model that explains these polarity differences is proposed that takes into account the asymmetry of the thermal properties of the two contacts combined with the change in energy deposition at the contacts between negative and positive biases. This asymmetry leads to pronounced energy deposition near the top interface under a positive bias, which depends only on the magnitude of the current. Under a negative bias, however, the energy deposition is much less pronounced at the top contact. These differences, combined with the differences in the heat sinking at both contacts, explain why it is generally easier to form devices with a positive voltage on the top contact when compared with a negative voltage.

\section{ACKNOWLEDGMENT}

We wish to thank Philips Research Laboratories (UK) for providing funding and the samples used in this work.

${ }^{1}$ A. M. Barnett and A. G. Milnes, J. Appl. Phys. 37, 4215 (1966).

${ }^{2}$ J. Hajto, A. E. Owen, A. J. Snell, P. G. LeComber, and M. J. Rose, Amorphous \& Microcrystalline Semiconductor Devices (Artech House, Boston, 1992), Vol. 2, p. 641.

${ }^{3}$ S. R. Ovshinsky, Phys. Rev. Lett. 21, 1450 (1968).

${ }^{4}$ J. F. Gibbons and W. E. Beadle, Solid-State Electron. 7, 785 (1964).

${ }^{5}$ C. Feldman and K. Moorjani, Thin Solid Films 5, R1 (1970).

${ }^{6}$ M. C. Gabriel and D. Adler, J. Non-Cryst. Solids 48, 297 (1982).

${ }^{7}$ J. M. Shannon and S. P. Lau, Electron. Lett. 35, 1976 (1999).

${ }^{8}$ R. G. Gateru, J. M. Shannon, and S. R. P. Silva, Mater. Res. Soc. Symp. Proc. 742, k.2.3 (2003)

${ }^{9}$ I. S. Osborne, J. Hajto, M. J. Rose, A. J. Snell, P. G. LeComber, and A. E. Owen, Mater. Res. Soc. Symp. Proc. 258, 1169 (1992).

${ }^{10}$ J. Hu, A. J. Snell, J. Hajto, and A. E. Owen, J. Non-Cryst. Solids 227, 1187 (1998).

${ }^{11}$ J. M. Shannon, R. G. Gateru, and E. G. Gerstner, Electron. Lett. 38, 249 (2002).

${ }^{12}$ J. Frenkel, Phys. Rev. 54, 647 (1938).

${ }^{13}$ K. J. B. M. Nieuwesteeg, M. van der Veen, and T. J. Vink, J. Appl. Phys. 74, 2572 (1993)

${ }^{14}$ R. A. C. M. M. van Swaaij, A. D. Annis, B. J. Sealy, and J. M. Shannon, J. Appl. Phys. 82, 4800 (1997).

${ }^{15}$ J. M. Shannon and C. H. Chua, Solid-State Electron. 47, 1903 (2003).

${ }^{16}$ K. J. B. M. Nieuwesteeg, M. van der Veen, T. J. Vink, and J. M. Shannon, J. Appl. Phys. 74, 2581 (1993).

${ }^{17}$ J. M. Shannon, S. P. Lau, A. D. Annis, and B. J. Sealy, Solid-State Electron. 42, 91 (1998). 